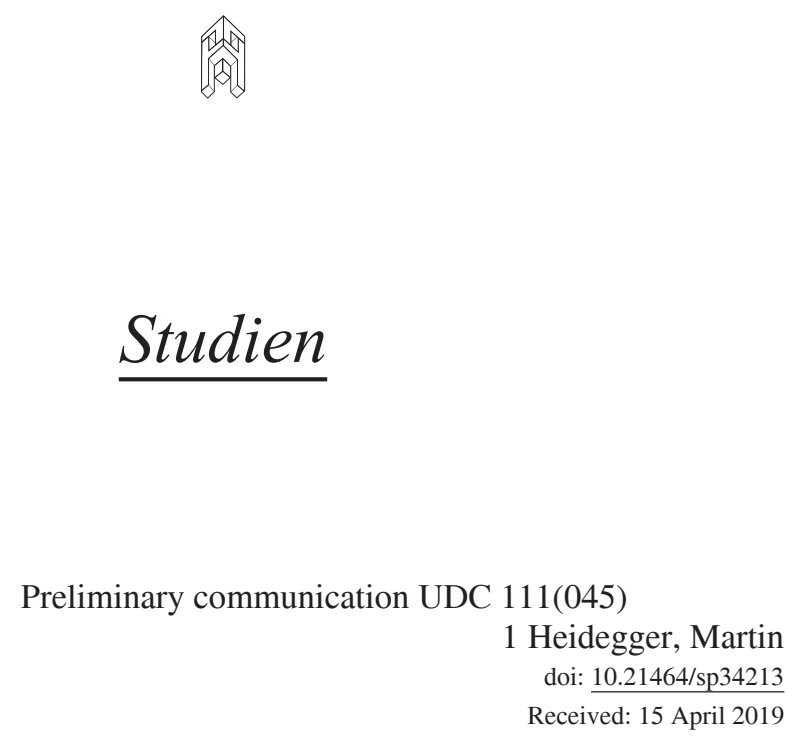

\footnotetext{
Biljana Radovanović, ${ }^{1}$ Goran Ružić ${ }^{2}$

Universität Niš, Philosophische Fakultät, Ćirila i Metodija 2, RS-18000 Niš

${ }^{1}$ biljana.radovanovic@filfak.ni.ac.rs, ${ }^{2}$ goran.ruzic@ filfak.ni.ac.rs
}

\title{
Heideggers Radikalisierung der kantischen Lehre von den mathematischen Schemata und der Zeit
}

\begin{abstract}
Zusammenfassung
Das Grundmotiv von Heideggers Interpretation der kantischen theoretischen Philosophie besteht darin, zu zeigen, dass Kants Lehre vom Schematismus und von der Zeit lediglich eine Vorstufe zur Problematik der Temporalität darstellt. In diesem Kontext analysieren wir Heideggers These von der transzendentalen Einbildungskraft als „Quelle“ der Zeit, beziehungsweise die mathematischen Schemata der Zeitreihe und des Zeitinhalts. Der notwendige Zusammenhang dieser Schemata mit den Kategorien der Quantität und Qualität, wie auch mit den Grundsätzen (den synthetischen Urteilen a priori) des reinen Verstandes, die Kant als „Axiome der Anschauung “ und "Antizipationen der Wahrnehmung " bezeichnet, erhöht die Komplexität unseres interpretativen Vorhabens. Zugleich muss die Einheitlichkeit der Zeit (als formale Anschauung) mit der Einheitlichkeit der ursprünglichen Apperzeption (Strukturen der Eigenwahrnehmung) in Verbindung gebracht werden, ebenso die durch die Sinne wahrgenommene Mannigfaltigkeit mit der Zeit als Form einer rein sinnlichen Anschauung. Die vergleichende Analyse der Ausgaben A und B der Kritik der reinen Vernunft ermöglicht uns, anhand von Heideggers Vorlesungen und Studien die Möglichkeiten einer ,,ontologisierten “ und phänomenologisch orientierten Interpretation der Grundannahmen der kantischen , Theorie des Erkenntnissubjekts" innerhalb des kantischen Texts aufzuspüren.
\end{abstract}

\section{Schlüsselwörter}

Transzendentalphilosophie, Schema, Raum, Zeit, Anschauung, Wahrnehmung, Verstand, Apperzeption

Nur mit Mühe können alle Stellen in Heideggers umfassendem Werk angeführt werden, die sich direkt mit der Analyse der kantischen theoretischen Philosophie beschäftigen, insbesondere mit der Problematik der Temporalität. Es besteht jedoch kein Zweifel, dass der Freiburger Philosoph mindestens drei seiner Studien der Betrachtung des Themas „Zeit“ bei Kant widmete, und zwar: Phänomenologische Interpretation von Kants Kritik der reinen Vernunft; Die Frage nach dem Ding. Zu Kants Lehre von den transzendentalen Grundsätzen; Kant und das Problem der Metaphysik. Der Stellenwert, den 
Kants theoretische Philosophie in Heideggers Opus einnimmt, kann über eine kritische Betrachtung der Vergangenheit bestimmt werden, d. h. über den hermeneutischen Ansatz bekannt als temporale Destruktion der Geschichte der westeuropäischen Metaphysik. Wenngleich das Verfahren der Destruktion negative Assoziationen hervorrufen kann, muss es als kritische Analyse der Konzepte und Inhalte gesehen werden, die der Wiederaneignung einer verzerrten und willkürlich interpretierten Tradition dienen soll. Sie steht nicht im negativen Verhältnis zur Vergangenheit selbst, sondern zu den heute vorherrschenden Deutungen der Vergangenheit (Geschichte der westeuropäischen Metaphysik), und zwar aufgrund der Reaktualisierung der ursprünglichen Erfahrungen in Bezug auf die philosophischen Grundbegriffe und die essentiellen philosophischen Fragen. Zeitlich betrachtet erfolgt die Destruktion im Rückschritt, beginnend mit Kants Lehre vom Schematismus und der Zeit als Vorstufe der Problematik der Temporalität über Descartes” „Ich denke, also bin ich" bis hin zu Aristoteles' Abhandlung über die Zeit (vgl. Heidegger 1993: 23-27). Nach Heideggers Einschätzung war Kant der erste, der sich „getrieben von den Phänomenen selbst" auf die Problematik der Temporalität hin zubewegte. Er wurde jedoch von der Dunkelheit, in welche der Schematismus ,in den Tiefen der menschlichen Seele“ gehüllt ist, daran gehindert, in den unverdeckten Bereich der Temporalität des Seins Eingang zu finden.

„Am Ende sind gerade die Phänomene, die in der folgenden Analyse unter dem Titel ,Temporalität ' herausgestellt werden, die geheimsten Urteile der , gemeinen Vernunft" als deren Analytik Kant das ,Geschäft der Philosophen“ bestimmt.“ (Heidegger 1993: 23)

Es gibt zwei Gründe, warum Kant die Einsicht in die Problematik der Temporalität verwehrt blieb: Zum einen wurde keine Verbindung zwischen der Zeit und der transzendentalen Apperzeption (dem „Ich denke“) hergestellt, zum anderen hat Kant die Ontologie des Da-seins nicht thematisiert, beziehungsweise, er ließ die Frage nach dem Sein außer Acht und lenkte damit die Analyse der Zeit in Richtung eines ontischen Verständnisses der Zeit, das ihre transzendentale Bestimmung nicht berücksichtigt. Den Ursprung der kantischen Versäumnisse erblickt Heidegger in Descartes' Hervorhebung der „absoluten Gewissheit“" des Cogito, die ihn vermeintlich der Thematisierung der Frage nach dem Sinn des Seins und der Verknüpfung der Subjektivität mit dieser Frage zu entheben schien. Nach der Destruktion der kantischen Lehre vom Schematismus und der Zeit ist eine Destruktion des ontologischen Primats des Cogito nötig. Schließlich ist das Endziel die Destruktion der temporalen Grundlage der antiken Ontologie (Aristoteles) und deren Interpretation des Seins als Vorhandensein. Im Zuge der Hinterfragung der überlieferten ontologischen Tradition erblickte Heidegger sowohl die Möglichkeit zu einer Konkretisierung der Frage nach dem Sinn des Seins, als auch deren Wiederholung und genealogische Fortführung. Die Erwähnung der Destruktion war unumgänglich, nicht nur aus geschichtlichen Gründen, sondern auch wegen der Feststellung des Stellenwerts der kantischen Lehre vom Schematismus und der Zeit innerhalb dieses phänomenologisch-hermeneutischen methodologischen Verfahrens. Nach dieser kurzen Einleitung richten wir unsere Aufmerksamkeit nun auf Heideggers Interpretation der kantischen Lehre vom Schematismus und der Zeit, oder genauer, von den mathematischen Schemata und der Zeit.

Transzendentale Schemata stellen für Kant ein Muster für das Wirken der reinen (produktiven und reproduktiven) Einbildungskraft in der Synthese elementarer und komplexer Gegebenheiten der Sinne dar, oder, mit Kants Worten: Transzendentale Schemata sind „Vorstellung[en] (...) von einem 
allgemeinen Verfahren der Einbildungskraft" (Kant 1976: A 140). Hier ist von den rein sinnlichen Anschauungen die Rede, die auch ein Modell für die empirische Synthese bieten.

„Die Schemate sind (...) nichts als Zeitbestimmungen [Kant verwendet Sperrungen, wir setzen die Ausdrücke kursiv - Anmerkung der Autoren] a priori nach Regeln, und diese gehen nach der Ordnung der Kategorien, auf die Zeitreihe, den Zeitinhalt, die Zeitordnung, endlich den Zeitinbegriff in Ansehung aller möglichen Gegenstände.“ (Kant 1976: A 145)

Die vier übergeordneten Kategorien (Quantität, Qualität, Relation und Modalität) stellen eigentlich eine Transposition der transzendentalen Schemata der Zeitreihe, des Zeitinhalts, der Zeitordnung und des Zeitinbegriffs dar. Über das transzendentale Schema kann gesagt werden, dass es einerseits eine zeitliche Bestimmung darstellt, andererseits einen „sinnlichen Begriff“. Die Kategorie besteht nämlich in der Einheit des transzendentalen Schemas, weswegen sie allgemeingültig ist, aber sie hat auch singulären Charakter, weil sie der von den Sinnen wahrgenommenen Einzelerscheinung ähnelt.

„Daher wird eine Anwendung der Kategorie auf Erscheinungen möglich sein, vermittelst der transzendentalen Zeitbestimmung, welche, als das Schema der Verstandesbegriffe, die Subsumtion der letzteren unter die erste vermittelt." (Kant 1976: A 139)

Im Hinblick auf die zwei Wege der transzendentalen Ableitung der Kategorien (in der Richtung von der Anschauung zum Begriff und, umgekehrt, vom Begriff zur Anschauung) wird auch die doppelte Funktion der rein sinnlichen Transposition erkennbar, die nichts anderes ist als eine Vorstellung von der Tätigkeit der Einbildungskraft, durch die ein Begriff verbildlicht wird. Das ist ein Schema.

„Die reine Versinnlichung geschieht als ein ,Schematismus‘. Die reine Einbildungskraft gibt Schema-bildend im vorhinein den Anblick (,Bild“) des Horizontes der Transzendenz.“ (Heidegger 1973: 88)

Hierbei muss das Problem der Relation zwischen Schema und Bild thematisiert werden, da auch Kant der Ansicht war, dass ,unseren reinen sinnlichen Begriffen nicht Bilder der Gegenstände, sondern Schemate zum Grunde“" liegen (Kant 1976: B 180). Das Bestehen von (empirischen) Bildern ist nur möglich, wenn ein Monogramm (eine Aufzeichnung) der reinen Einbildungskraft verfügbar ist, das ein Bild mit einem Begriff (mit einer allgemeinen Bestimmung) verknüpft, um den begriffenen Gegenstand zu vereinheitlichen. ${ }^{1}$ Wir unterscheiden das Bild, entstanden aus der empirischen Einbildungskraft, vom reinen Bild aller durch die Sinneswahrnehmung erfassten Gegenstände, genauer gesagt - von der Zeit. Ist das Bild ein Erzeugnis der empirischen Einbildungskraft, so ist ein reines Bild das Erzeugnis der transzendentalen Einbildungskraft, insofern ,ich die Zeit selbst in der Apprehension der Anschauung erzeuge" (Kant 1976: A 143). Heidegger geht davon aus, dass Kant den Begriff „Bild“ in drei verschiedenen, aber untereinander verbundenen Bedeutungen verwendet:

„... unmittelbarer Anblick eines Seienden, vorhandener abbildender Anblick eines Seienden und Anblick von etwas überhaupt.“(Heidegger 1973: 89)

Der Schematismus als sinnliche Transposition eines Begriffs (in ein Schema) soll auch die Relation zwischen Schema und Bild sichern, sowie die Relation

Wir können zwar ein beliebiges Dreieck zeichnen, nicht jedoch das Schema des Dreiecks, da dieses Schema - das Dreieck im Allgemeinen bzw. das Schema eines beliebigen Dreiecks der Begriff des Dreiecks ist, d. h. die Regel der Synthese reiner Formen im Raum. 
zwischen Begriff und Bild dank der vermittelnden Vorstellung, die Heidegger „Schema-Bild“ nennt und deren Gültigkeit er an den Beispielen empirischer Begriffsbestimmungen (Hund) und der rein mathematischen Bestimmung der Begriffe (Zahl und Dreieck) untersucht. Nach dieser Interpretation würde das Schema-Bild de facto ein Bild des Begriffs darstellen, welches als empirische Erscheinung eines Gegenstands dasselbe beinhaltet wie der Begriff. Unter Berücksichtigung der Art der Einheit (das Eine, das als das Mannigfache gilt) kann die empirische Erscheinung jedoch keine empirische Begriffsbestimmung erhalten. Mit dem Erfassen (Aufsammeln, Durchsuchen, Umfassen) der durch die Sinne gegebenen Mannigfaltigkeit eines Gegenstands in der Wahrnehmung erzeugt die Einbildungskraft ein Bild von dem Gegenstand, z. B. einem Hund, in dem der erfasste Gegenstand der Hund ist, weil er aus der Apprehension der Einbildungskraft gemäß dem Verfahren (Schema) zur Konstruktion der Gestalt des Hundes (Realisierung des Begriffs des Hunds) gebildet wurde, durch die Synthese der Apprehension einer über die Sinne gegebenen Mannigfaltigkeit.

„Der Begriff vom Hunde bedeutet eine Regel, nach welcher meine Einbildungskraft die Gestalt eines vierfüßigen Tieres allgemein verzeichnen kann, ohne auf irgendeine einzige besondere Gestalt, die mir die Erfahrung darbietet, oder auch ein jedes mögliche Bild, was ich in concreto darstellen kann, eingeschränkt zu sein.“ (Kant 1976: A 141)

Zwei Hunde sind zum Beispiel zwei in Bezug auf die räumliche, äußere ( $a u$ ßereinander), parallele (nebeneinander) Einheit, aber sie sind zwei Hunde auch in Bezug auf die Gleichheit des synthetischen Vorgangs im Zuge der Apprehension der durch die Sinne gegebenen Mannigfaltigkeit. Hier ist nicht die Rede von einer kategorialen Einheit (Kategorie der Quantität), sondern von der Einheit einer Mannigfaltigkeit aufgrund der Synthese eines Gegenstands bzw. aufgrund der Vielheit von Synthesen derselben Gegenstände. Die Einheit der Mannigfaltigkeit im Raum ist irrelevant, parallel und äußerlich, im Unterschied zur zeitlichen Einheit, in der etwas Mannigfaltiges sukzessiv (nacheinander) erscheint. Ist es in Bezug auf zwei Gegenstände völlig willkürlich (irrelevant), ob beide Hunde etwas besitzen, was man als vier Beine bezeichnen könnte, so stellt das Erkenntnissubjekt (der Mensch) die Gleichheit (zweier Hunde) gemäß der Einheit der ursprünglichen Apperzeption her. Die extensive (und intensive) Mannigfaltigkeit der sinnlich gegebenen Gegenstände der Anschauung muss synthetisch vereinheitlicht werden (im Sinne der Einheit der Erkenntnisgegenstände). Das Vermögen, das diese Einheit der Mannigfaltigkeit von Wahrnehmungen (und Anschauungen) ermöglicht, wird bei Kant die transzendentale oder ursprüngliche Apperzeption genannt. Diese Einheit der sinnlich gegebenen Mannigfaltigkeit wird mittels der Vorstellung einer Verbindung, d. h. vermittelst des „Ich denke“, das alle meine Vorstellungen begleiten können muss, hergestellt, allerdings nicht als (eigene) Selbsterkenntnis, sondern als Verstandesvorstellung von der Selbsttätigkeit eines Denksubjekts.

Heidegger ist der Meinung, dass mathematische Schema-Bilder näher (adäquater) an ihren Begriff herankommen als die Schema-Bilder empirischer Gegenstände oder, anders gesagt, das mathematische Schema-Bild kommt der Einheit des Begriffs nahe bzw. der Allgemeinheit der Einheit (Heidegger 1976: 322-334, Kapitel 26).

„Hieraus wird erst das Wesentliche des Schema-Bildes deutlich: es hat seinen Anblickcharakter nicht nur und zuerst aus seinem gerade erblickbaren Bildgehalt, sondern daraus, daß es und wie es aus der in ihrer Regelung vorgestellten möglichen Darstellung herausspringt und so gleichsam die Regel in die Sphäre der möglichen Anschaulichkeit hineinhält.“ (Heidegger 1973: 96) 
Über die Analyse des bildhaften Charakters des Schema-Bildes will Heidegger zeigen, dass die sinnliche Transposition der Begriffe eine besondere Form der Bilderzeugung darstellt. Die Worte des Freiburger Philosophen klingen fast tautologisch, wenn er behauptet, dass die sinnliche Transposition im Sinne einer unmittelbaren empirischen Anschauung des Gegenstands und der Hervorbringung empirischer Gestalten nur auf der Basis einer sinnlichen Transposition von Begriffen (= eines Schematismus) möglich ist, bzw. dass jede begriffliche Vorstellung ihrem Charakter nach einem Schematismus gleicht, als Methode und Verfahren zur Umwandlung eines Begriffs in ein Bild. Ein transzendentales Schema ist jedoch weder Bild noch Begriff, sondern ,etwas Drittes", was in sich selbst sowohl den Charakter einer Erscheinung (Sinnlichkeit), als auch den Charakter einer Kategorie (Begrifflichkeit, Rationalität) trägt. Es ist also nicht die Rede von einer Mischung aus Sinnlichem und Rationalem als einer Art a posteriori-Synthese oder von irgendeinem Verfahren zum Auffinden eines Bildes als sinnlicher Anschauung der Kategorien, sondern es bedarf einer Hinwendung zu den Bildern rein sinnlicher Begriffe, d. h. der Begriffe der Arithmetik und Geometrie als mathematischer Begriffe, die verallgemeinerte Konstruktionen in Raum und Zeit darstellen. Mathematische Begriffe im Raum sind eigentlich Abstraktionen einzelner Dreiecke, Quadrate, Rechtecke in Form von Bildern, d. h. Anschauungen, während sie in der zeitlichen Dimension Abstraktionen einzelner Zahlen als Anschauungen in der Zeit darstellen, weshalb für sie ein Bild mithilfe eines reinen Schemas mathematischer Begriffe konstruiert werden kann. Es ist im Sinne von Kants Philosophie, wenn man sagt, dass das Schema eines reinen Verstandesbegriffs lediglich eine reine Synthese darstellt (Kant 1976: A 142). Kategorien als die allgemeinsten Begriffsbestimmungen beinhalten nichts Sinnliches (z. B. ein Bild oder eine sinnliche Anschauung), sondern nur ein reines Schema. Ein rein sinnlicher Begriff (z. B. der Begriff einer Zahl oder einer geometrischen Figur) kann andererseits entweder ein Bild oder eine Anschauung oder Konstruktion beinhalten. Die größte Herausforderung stellt für uns Heideggers Feststellung dar, dass die Zeit als reines Bild eigentlich ein Schema-Bild darstellt, nicht nur eine ,,den reinen Verstandesbegriffen gegenüberstehende Anschauungsform“ (vgl. Heidegger 1973: 100; Heidegger 1976: 275). Leider arbeitet Heidegger die Gedanken zur Zeit (reines Bild) als Schema-Bild nicht bis zum Schluss auf eine klare und eindeutige Weise weiter aus, sondern er fasst nur die Stellen aus der Kritik der reinen Vernunft (A 138-B 187) zusammen, wo von den transzendentalen Schemata der Kategorien (Quantität, Qualität, Relation und Modalität), sowie den Schemata ihnen untergeordneter Kategorien die Rede ist. Zugleich deutet er an, dass die transzendentale Einbildungskraft eine Quelle der Zeit sei. ${ }^{2}$ Die transzendentale Einbildungskraft als Erkenntnisvermögen ,,produziert“ vermittels der reinen Synthese transzendentale Schemata und stellt damit die Einheit der rein sinnlichen Anschauung (Zeit) und des reinen Verstandesbegriffs (Kategorie) her. Heidegger ist jedoch der Ansicht, dass die transzendentale Einbildungskraft nicht den zentralen Platz in der Lehre vom transzendentalen Schematismus einnimmt, sondern in der transzendentalen Deduktion der Verstandesbegriffe $\mathrm{zu}$ verorten ist. Weit mehr als für die figurative und intellektuelle Synthese des Verstandes (aus der Ausgabe B) interessiert sich Heidegger für die

2

Die Rede ist von der Analyse des transzendentalen Schemas der Substanzkategorie (Persistenz dessen, was in der Zeit real ist), das er zu Recht mit der ersten Analogie der
Erfahrung in Verbindung bringt, bzw. es werden die Konsistenz (der Substanz) und die Dauer (der Zeit) miteinander in Verbindung gebracht. 
reinen Synthesen der transzendentalen Einbildungskraft (Apprehension, Reproduktion, Rekognition) aus der A-Ausgabe der Kritik der reinen Vernunft, welcher folglich auch der Vorzug gegeben wird (vgl. Heidegger 1977: 339359). Vielleicht wäre es besser, in der Analyse transzendentaler Schemata der reinen Kategorien (diese Schemata sind die Zeitreihe, der Zeitinhalt, die Zeitordnung und der Zeitinbegriff) von der Analyse einzelner transzendentaler Schemata der mathematischen Kategorien auszugehen. So würden wir erkennen, dass im transzendentalen Schema der Zeitreihe bereits die Quantitätskategorien (Einheit, Vielheit, Allheit) und das Axiom der Anschauung als Prinzip des reinen Verstandes und als synthetischer Satz a priori enthalten sind, der folgendermaßen lautet:

„[D]ie Erscheinungen sind insgesamt Größen, und zwar extensive Größen.“ (Kant 1976: A 162; siehe auch Heidegger 1984: 197)

Ein transzendentales Schema der Zeitreihe würde Folgendes umfassen: a) eine extensive ungeteilte Zeiteinheit; b) eine Vielheit von extensiven ungeteilten gleichen Zeiteinheiten; ${ }^{3}$ c) die Allheit derselben extensiven, ungeteilten Zeiteinheiten, die der Anzahl der Zeiteinheiten entspricht, was nichts anderes als eine Synthese (Verfahren) der transzendentalen Einbildungskraft in Form einer Aneinanderreihung (aggregatio) derselben Zeiteinheiten bedeutet. Die Zeit als Zeitreihe stellt eine Aneinanderreihung der extensiven einheitlichen Zeitintervalle dar (Extensität der Zeit), d. h. es ist die Rede vom transzendentalen Schema der Extensität der Zeitreihe. Das Syntagma „das mannigfaltige Gleichartige" beschreibt bei Kant eine Vielheit gleicher Einheiten als zahlenmäßiger Elemente, beziehungsweise, er spricht von der Synthesis der gleichen Vielheit - von der mathematischen Synthesis. Der Unterschied zwischen den mathematischen transzendentalen Schemata (Zeitreihe, Zeitinhalt) und den dynamischen transzendentalen Schemata (Zeitordnung, Zeitinbegriff) liegt darin, dass die erstgenannten (mathematische Schemata) apriorische Modelle der Ordnung beim Verfahren der Synthesis der Einbildungskraft im Prozess der Verknüpfung einer gleichartigen Vielheit darstellen (coniunctio qua compositio), während die letztgenannten (dynamische Schemata) im Prozess der Verknüpfung einer verschiedenartigen Vielheit (coniunctio qua nexus) tätig werden. Wenngleich bei beiden mathematischen transzendentalen Schemata die Synthesis einer gleichartigen Vielheit zum Zuge kommt, handelt es sich bei der Zeitreihe um eine Aggregation (Extension) zeitlicher und räumlicher Intervalle, beim Zeitinhalt um eine Koalition (Intension) zeitlicher und räumlicher Intervalle (Kant 1976: B 201-202; mit Sternchen markierte Fußnote auf Seite 216). Des Weiteren setzt Kant häufig Zeit und Raum mit der Zeitund Raumanschauung gleich, wobei er die Identität des Anschauungsgegenstands und der Anschauung voraussetzt. Diese Gleichsetzung hat zur Folge, dass Raum und Zeit nur dann existieren, wenn sie von der transzendentalen Einbildungskraft mittels der Synthesis der gleichartigen Vielheit (compositio) und der verschiedenartigen Vielheit (nexus) zeitlicher und räumlicher Intervalle geschaffen wurden. Als Gegenstand der Anschauung treten Zeit und Raum in der Synthesis auf, da wir

„... ohne sie [die Synthesis der Apprehension, Anm. der Autoren] (...) weder die Vorstellungen des Raumes, noch der Zeit a priori haben können [würden]: da diese nur durch die Synthesis des Mannigfaltigen, welches die Sinnlichkeit in ihrer ursprünglichen Rezeptivität darbietet, erzeugt werden können. Also haben wir eine reine Synthesis der Apprehension.“ (Kant 1976: A 99-100)

Die Synthesis der Apprehension erfolgt sowohl a priori, als auch a posteriori, was anhand zahlreicher Stellen der A- und B-Ausgabe der Kritik der reinen 
Vernunft belegt werden kann, Stellen, die sich gegenseitig nicht ausschließen. ${ }^{4}$ Die dreifache Synthesis der transzendentalen Einbildungskraft (Apprehension, Reproduktion, Rekognition) sollte man nicht als drei Synthesen verstehen, sondern als eine Synthese der Mannigfaltigkeit, die bei einer simultanen Rekognition (Identifikation) apprehendiert wird (Heidegger 1973: 171). Es wäre völlig fehl am Platz, sowie logisch und zeitlich unmöglich, hierbei von einer Sukzession dreier Synthesen zu sprechen, denn im Endeffekt würde dies bedeuten, dass die angebliche Aufeinanderfolge der Synthesen innerhalb eines Zeitintervalls stattfindet, was keinen Sinn ergibt. ${ }^{5}$ Im Rahmen der Darstellung des transzendentalen Schemas der Zeitreihe haben wir den Ausdruck „Extensität der Zeit“ verwendet, der auf den ersten Blick womöglich ungewöhnlich wirkt. Schließlich spricht man oft über die Zeit so, als ob sie etwas wäre, das sich durch die Addition der Zeitintervalle ,ausdehnt“" und mit dem Raum ,verwachsen“ ist.

„Ebenso ist es auch mit jeder auch der kleinsten Zeit bewandt. Ich denke mir darin nur den sukzessiven Fortgang von einem Augenblick zum anderen, wo durch alle Zeitteile und deren Hinzutun endlich eine bestimmte Zeitgröße erzeugt wird.“ (Kant 1976: A 163)

Mathematische Begriffe sind Konstruktionen im Raum (z. B. Abstraktionen einzelner Dreiecke oder Rechtecke als Bilder $=$ Anschauungen) und in der Zeit (Abstraktionen einzelner Zahlen, z. B. 3 und 7 als Anschauungen in der Zeit). Mittels reiner Schemata mathematischer Begriffe kann jeweils ein Bild für ihre Konstruktion gefunden werden. Kants Formulierung von Axiomen der Anschauung in der Ausgabe B ist zu ungenau und besagt, dass ,,[a]lle Anschauungen (...) extensive Größen“" sind (Kant 1976: B 202). Die Formulierung aus der Ausgabe A ist weitaus präziser, da sie darauf hinweist, dass die Extensität eines Gegenstands der Anschauung der wesentliche Bestimmungsgrund jeder Erscheinung ist. Dabei ist klar, dass alle Erscheinungen Zeitreihen darstellen und (alle Erscheinungen) als Anschauungsgegenstände extensive Größen repräsentieren, die mittels einer Aggregation einer gleichartigen Vielheit in der Zeitreihe entstehen. Unter der Form der Erscheinung versteht Kant die Möglichkeit zur Herstellung bestimmter Verhältnisse innerhalb der Mannigfaltigkeit einer Erscheinung, während er unter der Materie (dem Inhalt) der Erscheinung dasjenige versteht, was der Empfindung in der Erscheinung entspricht (vgl. Kant 1976: A 20, Heidegger 1984: 198). Die Synthesis der Apprehension in der transzendentalen Einbildungskraft sieht Heidegger als jene Synthesis, welche die Zeit formt und er schließt daraus, dass diese

Kant verwendet den Ausdruck „das mannigfaltige Gleichartige“, wobei es in diesem transzendentalen Schema um eine Vielheit gleicher Teile geht. Der Begriff „Mannigfaltigkeit" wird von Kant (gemeinsam mit den Paronymen „mannigfaltig“ und „Mannigfaltiges") hauptsächlich zur Beschreibung des Prozesses der Begriffsbildung verwendet.

4

So ist, zum Beispiel, in der A-Ausgabe die Rede von der Synthese der Apprehension, die ,,auch a priori d. i. in Ansehung der Vorstellungen, die nicht empirisch sind, ausgeübt werden“" muss (A 99), von der ,reine[n] Synthesis der Apprehension“ (A 100), während es in der B-Ausgabe heißt: ,Zuvörderst merke ich an, daß ich unter der Synthesis der
Apprehension die Zusammensetzung des Mannigfaltigen in einer empirischen Anschauung verstehe, dadurch Wahrnehmung, d. i. empirisches Bewußtsein derselben, (als Erscheinung) möglich wird.“ (B 160) 5

Der erste Grundsatz des reinen Verstandes bezüglich der Tätigkeit der reproduktiven und produktiven Einbildungskraft ist das Axiom der Anschauung als Grundlage und Bedingung der Möglichkeit aller mathematischen Axiome und aller Konstruktionen geometrischer rein sinnlicher Begriffe (Kants Ausdruck in A 140), sowie als Gattungsbegriff der mathematischen Schemata der Zeitreihe und des Zeitinhalts. 
Einbildungskraft einen rein zeitlichen Charakter hat (vgl. Heidegger 1973: 174-175). Der Zeitmodus, auf den sich die Synthesis der Apprehension bezieht, ist die Gegenwart. Dies gilt auch für die Synthesis der Rekognition, während die Synthesis der Reproduktion demjenigen zugewandt ist, was war, also dem in zeitlicher Hinsicht Früheren. Die Frage nach der Produktivität der reproduktiven Einbildungskraft ist mehr rhetorisch als essentiell, denn es besteht kein Zweifel, dass in der Synthesis der Reproduktion eine Gestalt ohne deren Anwesenheit in der Anschauung produziert wird. Heidegger meint, dass der dritte Modus der Synthesis (die reine Rekognition im Begriff) den „Horizont“ der Zukunft formt (vgl. Heidegger 1977: 359). Eine vorsichtigere Formulierung wäre vielleicht, dass durch eine reine Synthese der Rekognition im Begriff im Voraus die Möglichkeit zur Identifikation eines anschaulich gegebenen Gegenstands gegeben wird. Andernfalls könnte angenommen werden, dass die Rekognition eine ,nachträgliche“ Synthese darstellt, welche sukzessive ,nach“ der Apprehension erfolgt, was unmöglich ist. An anderer Stelle scheint es, als ob Heidegger, wenn er von der Synthese der Rekognition im Begriff spricht (von der Vorstellung der Einheit des Verschiedenartigen), seine Gedanken spezifizieren und sich selbst korrigieren würde:

„Sie erkundet im voraus und ist, hindurchspähend“ auf das, was im vorhinein als das Selbige vorgehalten sein muß, damit die apprehendierende und die reproduzierende Synthesis überhaupt einen geschlossenen Umkreis von Seiendem vorfinden können, innerhalb dessen sie das, was sie beibringen und antreffen, als Seiendes gleichsam anbringen und hinnehmen können.“ (Heidegger 1973: 180)

Nach Heideggers scheinbar beruhigenden Worten über die Tätigkeit der Synthese der Rekognition im Begriff, die durch die Zweideutigkeit des Begriffs $a$ priori (vorher und im Voraus) ihren reinen Aspekt hervorheben, beziehen wir eine Argumentationsebene, auf der sich der Freiburger Philosoph explizit für den futuristischen Charakter der Synthese der Rekognition entscheidet, wenn nach seiner Vermutung ,in dieser scheinbar ganz zeitabgewandten Kantischen Analyse der reinen Synthesis im Begriffe gerade das ursprünglichste Wesen der Zeit zum Vorschein“ kommt: „daß sie sich primär aus der Zukunft zeitigt“ (vgl. Heidegger 1973: 181). Es besteht kein Zweifel, dass es sich hierbei um Heideggers authentische Worte handelt. In Anbetracht der Tatsache, dass in der Ausgabe A der Kritik der reinen Vernunft in den Passagen von A 103 bis A 110 unter der Überschrift „Von der Synthesis der Rekognition im Begriffe“ keine aus der Zukunft ,,zeitigende Zeit“" (reflexive Temporalisierung) genannt wird, ist es fraglich, ob Kant mit Heideggers Worten einverstanden wäre. Zudem kann man Kants Worten in diesem Werk keine Mehrdeutigkeit unterstellen, aufgrund welcher der Interpret (Heidegger) eine Korrektur des Texts vornehmen könnte, um uns angeblich eine ,richtige“ und authentische Deutung des kantischen Textes anzubieten, was vermutlich eine „sinngemäße“, nicht aber eine „wortgetreue“ Richtigkeit bedeuten soll. Hier sind wir aber noch immer nicht am Ende, da Heidegger aufgrund des „Hineinlesens“ seiner eigenen Philosophie in deren Kants schlussfolgert:

„... die transzendentale Einbildungskraft ist die ursprüngliche Zeit.“ (Heidegger 1973: 181)

Das Bestehen auf dem zeitlichen Charakter der transzendentalen Einbildungskraft impliziert auch die nachfolgende These, nämlich jene, dass die Zeit (als solche), als reine Selbstaffektion den Charakter des Selbst hat. Dieser These werden wir etwas mehr Aufmerksamkeit schenken, vor allem, weil Kant (tatsächlich) davon spricht, dass das Gemüt von der eigenen Tätigkeit affiziert wird, indem es die eigene Anschauung in sich selbst setzt. Hier die entsprechende Stelle: 
„Nun ist das, was, als Vorstellung, vor aller Handlung irgend etwas zu denken, vorhergehen kann, die Anschauung, und, wenn sie nichts als Verhältnisse enthält, die Form der Anschauung, welche, da sie nichts vorstellt, außer so fern etwas im Gemüte gesetzt wird, nichts anderes sein kann, als die Art, wie das Gemüt durch eigene Tätigkeit, nämlich dieses Setzen ihrer Vorstellung, mithin durch sich selbst affiziert wird, d. i. ein innerer Sinn seiner Form nach." (Kant 1976: B 67-68)

Zunächst muss betont werden, dass der Sinn von Kants Meinung, dass das Gemüt durch seine eigene Tätigkeit affiziert werde, darin liegt, dass es die Anschauung eines äußeren Sinnes in sich selbst setzt und damit den inneren Sinn (die Zeit) zur Anschauung anregt. Die Vorstellungen des äußeren Sinnes werden ins Subjekt gesetzt, indem das Subjekt selbst diese Vorstellungen setzt und somit eine Anschauung erzeugt und seinen eigenen inneren Sinn affiziert, denn, wie Heidegger richtig hervorhebt: alle Vorstellungen „fallen“ in die Zeit (sowohl des inneren wie des äußeren Sinnes), weshalb die Bestimmung der Zeit als reiner universeller Anschauung und intersubjektiv verbindlicher Form der Sinnlichkeit berechtigt ist. Die kantische Theorie von der Idealität des äußeren und inneren Sinnes gründet auf der Prämisse, dass zur Anschauung, d. h. zur Zeit als Anschauungsform, a priori die Extension (Ausdehnung), die Bewegung als Ortswechsel und die Gesetze gehören, nach denen die (extensiven und intensiven) Änderungen erfolgen müssen (Antriebskräfte). Alle Vorstellungen (des äußeren und inneren Sinnes) sind Erscheinungen und gehören als solche der Zeit an. ${ }^{6}$ Die Anschauung des erkennenden Subjekts ist sinnlich, endlich, subjektiv, aber auch apriorisch. Gerade die Sinnlichkeit verfügt über apriorische Formen - Raum und Zeit. Außerdem ist die Anschauung ein Bestandteil der Erkenntnis, sie ist einzeln und bezieht sich unmittelbar auf einen Gegenstand. Ein zweiter konstitutiver Aspekt der Erkenntnis ist der Begriff, der sich mittels Anschauung auf Gegenstände bezieht.

„Mit anderen Worten, unsere Anschauungen stellen unserer Erkenntnis lediglich Vorstellungen von Verhältnissen zur Verfügung. Hierbei ist Vorsicht geboten und Kants Worte sollten ad litteram verstanden werden, denn Kants Worte, alles “ was in unserer Erkenntnis Teil der Anschauung ist (...) bedeuten nicht, dass hierbei ,alles ' bereits die Erkenntnis an sich ist, sondern nur ein Teil davon - einer ihrer grundlegenden Bestandteile." (Todorović 2004: 138)

Die Zeit als reine Sinnesanschauung treibt sich selbst an und antizipiert nicht nur das Erscheinungsbild der Sukzession, sondern auch der Gleichzeitigkeit (Simultaneität) und der Permanenz (Sukzession, Simultaneität).

„Die Zeit ist als reine Selbstaffektion nicht eine wirkende Affektion, die ein vorhandenes Selbst trifft, sondern als reine bildet sie das Wesen von so etwas wie Sich-selbst-angehen. “ (Heidegger 1973: 183)

Die Frage der Selbstaffektion kann auch anders gestellt werden: Wie kann ein erkennendes Subjekt auf immanente (innere) Art und Weise sich selbst wahrnehmen? ${ }^{7}$ Es stellt sich heraus, dass die Zeit als reine Selbstaffektion die

Wenn wir sagen, dass alle Sinnesobjekte einfach nur Vorstellungen sind, bedeutet dies, dass diese Objekte selbst keine Dinge an sich darstellen, sondern Erscheinungen, also nehmen wir auch uns selbst (das transzendentale Subjekt) als Erscheinung wahr. „Um eine Antwort auf diese Fragen $\mathrm{zu}$ finden, muss Kant die Struktur unserer Erkenntnis aufzeigen, die als Anschauung und Begriff notwendigerweise eine (bewusste) Vorstellung ist." (Todorović 2004: 138 - unsere Übersetzung, Anm. der Autoren)
„Die Zeit ist nur so reine Anschauung, daß sie von sich aus den Anblick des Nacheinander vorbildet und diesen als solchen auf sich als das bildende Hinnehmen $z u$-hält. Diese reine Anschauung geht mit dem in ihr gebildeten Angeschauten sich selbst an, und zwar ohne Beihilfe der Erfahrung. Die Zeit ist ihrem Wesen nach reine Affektion ihrer selbst." (Heidegger 1973: 183) 
reine Rezeptivität darstellt (Form der Sinnlichkeit). Alle Vorstellungen, seien sie durch den Einfluss äußerer Erscheinungen oder aus inneren Ursachen entstanden, unterliegen als Modifikationen des Bewusstseins der formellen Bedingung des inneren Sinnes - der Zeit, in der sie sich verbinden, sammeln, verschmelzen und in bestimmte Beziehungen miteinander eingehen, egal ob sie empirisch oder a priori entstanden sind. Die besondere Natur der Endlichkeit des transzendentalen Subjekts bezeichnet Heidegger mit einem Ausdruck, mit dem Kant wohl kaum einverstanden gewesen wäre: Er bezeichnet sie als rein sinnliche Vernunft, wobei er zugleich die Heterogenität der reinen Sinnlichkeit und der reinen Vernunft hervorhebt. Ein Wegweiser für die Hinterfragung des zeitlichen Charakters des Selbst ist Kants explizite Anführung der transzendentalen Idealität der Zeit (d. h., wenn man von den subjektiven Bedingungen der Sinnlichkeit abstrahiert, ist die Zeit gar nichts). Die Untersuchung der Endlichkeit und Zeitlichkeit des Erkenntnissubjekts sollte nicht als Heideggers „Abenteuer“ betrachtet werden, sondern als Knotenpunkt in Kants Text. Der Freiburger Philosoph beginnt die Analyse des (inter)subjektiven Charakters der Zeit mit einer Klärung der nicht besonders klaren kantischen Formulierung „Begriff der Vorstellung von Gegenständen“. ${ }^{8}$ Hier ist die Rede von der Gegenständlichkeit, wobei die „Vergegenständlichung" notwendigerweise von der Zeit affiziert wird. Wer affiziert wen: Die Zeit den Gegenstand, oder der Gegenstand die Zeit? Die reine Anschauung, die Zeit, affiziert sich selbst, wenn sie vom Gegenstand (von der Vorstellung) angeregt wird. Wie soll dann die Rezeptivität der Sinnlichkeit mit der Vorstellung von der Zeit als Selbstaffektion in Einklang gebracht werden, die demnach eine Tätigkeit impliziert? Vielleicht so, dass die Zeit als apriorische Form der Sinnlichkeit auch die Struktur der Subjektivität prägt. Ist das Bewusstsein nämlich durch seine eigene Tätigkeit affiziert, ist dies ein Beweis für die These, dass Aktivität den primären Aspekt des Bewusstseins darstellt. Den Sinn (ob äußerer oder innerer) sieht Heidegger als ,endliche Anschauung“. Die Form dieses Sinnes ist von Rezeptivität gekennzeichnet, von der angenommen wird, dass sie im reinen Selbst entstehen muss. Heißt dies, dass Rezeptivität und Selbstaffektion ein und dasselbe sind? ${ }^{9}$ Die Zeit ist jedenfalls nichts, was sich neben der transzendentalen Apperzeption im Gemüt befände, sondern sie macht durch die Position in der reinen Apperzeption das Gemüt aus. Kants Worte in der A-Ausgabe der Kritik der reinen Vernunft von der Zeit, ,die sich nicht verläuft“, „die selbst unwandelbar und bleibend ist“", die „bleibt und nicht wechselt“, sprechen für eine Analogie des „Ichs" als Korrelat all unserer Vorstellungen und der Zeit als desjenigen, das bleibt und nicht vergeht (vgl. Heidegger 1973: 186). Kants Aussagen über die Zeit als dasjenige, was „bleibt und beharrt“ sind transzendentale Bestimmungen, in denen die reine Apperzeption (an der Beständigkeit festhaltend) über die Korrelate der Persistenz und Subsistenz den Horizont des Selbst formt. „Bleibt und beharrt“ bezieht sich nicht auf die Atemporalität (Nichtzeitlichkeit), sondern die Bestimmungen beziehen sich auf das „Ich“, wenn dieses „Ich“ die Gegenständlichkeit formt, bzw. wenn es zeitlich ist und die Eigenschaften eines endlichen Selbst besitzt. Dass Kant der transzendentalen Apperzeption und der theoretischen Vernunft den zeitlichen Charakter aberkennt, zeigt nur, dass diese keiner Zeitform unterliegen. Ähnliche die Zeit betreffenden Prädikate bedeuten nicht, dass die Zeit nicht „,in der Zeit“ (in sich selbst) liegt, sondern dass die Zeit als Selbstaffektion eine Sukzession in Form einer „Aneinanderreihung gegenwärtiger Momente“ (Augenblicke) als Grenze zwischen der vergangenen und der zukünftigen Zeit ermöglicht. ${ }^{10}$ Die Zeit ist eine Form des inneren (und äußeren) Sinnes bzw. der Anschauung 
unserer selbst und unserer inneren Zustände. Um die Reihe in ihrer Ganzheit und Totalität in der Zeit zu setzen, muss es möglich sein, den Augenblick festzuhalten und dabei nicht den allgemeinen Charakter der Zeit als ununterbrochene (kontinuierliche) Bewegung und Vergehen aus dem Blick zu verlieren. Genau das wird durch die ursprüngliche, aber synthetische Tätigkeit der transzendentalen Apperzeption gesichert. Heidegger stellt fest, dass das „Ich denke“, das als Vorstellung alle meine anderen Vorstellungen begleiten können muss, nicht der Zeit entgegengesetzt, sondern ihr gleichgestellt ist. Die Position der reinen Apperzeption korreliert dabei mit den Vorstellungen des äußeren und inneren Sinnes. ${ }^{11}$

So wie wir zuvor das transzendentale Schema der Zeitreihe mit der Kategorie der Quantität und den Axiomen der Anschauung in Verbindung gebracht haben, verbinden wir nun auch das transzendentale Schema des Zeitinhalts mit der Kategorie der Qualität und den Antizipationen der Wahrnehmung. Im Rahmen des ersten synthetischen Grundsatzes a priori (Axiome der Anschauung) hatten wir es mit der Darstellung der sinnlich gegebenen Erscheinungen zu tun, die sich, in Bezug auf ihre Anschauung, als extensive Größen herausgestellt haben. Im zweiten synthetischen Grundsatz a priori (Antizipationen der Wahrnehmung) ist ebenfalls die Rede vom Auftreten sinnlich gegebener Erscheinungen, aber nicht entsprechend ihrer Anschauung, sondern entsprechend ihrer Wahrnehmung:

„In allen Erscheinungen hat die Empfindung, und das Reale, welches ihr an dem Gegenstande entspricht, (realitas phaenomenon) eine intensive Größe, d. i. einen Grad.“ (Kant 1976: A 166, Fußnote 5)

\section{In der Ausgabe B finden wir folgende Formulierung vor:}

„In allen Erscheinungen hat das Reale, was ein Gegenstand der Empfindung ist, intensive Größe, d. i. einen Grad.“ (Kant 1976: B 207)

Nachdem wir uns diese Worte Kants zu Gemüte geführt haben, können wir nun unsere eigene Formulierung anbieten: Alle sinnlich gegebenen Erscheinungen sind hinsichtlich ihrer Wahrnehmung intensive Größen. Um uns dem

„Ganz generell können wir drei wichtige Strukturaspekte der Vorstellung unterscheiden: das Vorstellende als Subjekt (nicht im Sinne eines Trägers oder Vehikels) der Vorstellung, das als Objekt Vorgestellte als Gegenstand der Vorstellung (nicht als dasjenige, was ihr in der Außenwelt entspricht) und der Akt der Vorstellung an sich." (Todorović 2004: 138)

9

Heidegger sagt nämlich, dass ,[d]ie reine Selbstaffektion (...) die transzendentale Urstruktur des endlichen Selbst als eines solchen" gibt (Heidegger 1973: 185).

10

Im Paragraphen 32 von Kant und das Problem der Metaphysik bezieht sich Heidegger auf die Pölitz-Ausgabe der Vorlesungen zur Metaphysik (zur rationalen Psychologie), in der die bildende Kraft der transzendentalen Einbildungskraft als Vermögen zur Erzeugung einer Vorstellung von der vergangenen, jetzigen und zukünftigen Zeit definiert wird, und zwar: ,1. aus dem Vermögen der Abbildung, welches Vorstellungen der gegenwärtigen Zeit sind: facultas formandi; 2. aus dem Vermögen der Nachbildung, welches Vorstellungen der vergangenen Zeit sind: facultas imaginandi; 3. aus dem Vermögen der Vorbildung, welches Vorstellungen der zukünftigen Zeit sind: facultas praevidendi.“ (Heidegger 1973: 168-169)

11

Todorović ist der Meinung, dass sich das Phänomen des Vor-stellens aus „Setzen“ und „Besetzen“ zusammensetzt, wobei unter diesem „Setzen“ die Konstitution der Vorstellung (als solcher, als ,Vorstellung überhaupt") verstanden wird. Die Zeit ist hierbei ,der Grund als formale Bedingung der Art und Weise, auf die wir in unserem Gemüt (...) Vorstellungen als Vorstellungen setzen" (Todorović 2004: 140). 
transzendentalen Schema des Zeitinhalts anzunähern, müssen wir neben der „Antizipationen der Wahrnehmung“ mit dem ,anderen Auge“ einen Blick auf die Kategorie der Qualität werfen, über die Kant Folgendes sagt:

„Realität ist im reinen Verstandesbegriffe das, was einer Empfindung überhaupt korrespondiert; dasjenige also, dessen Begriff an sich selbst ein Sein (in der Zeit) anzeigt; Negation, dessen Begriff ein Nichtsein (in der Zeit) vorstellt. Die Entgegensetzung beider geschieht also in dem Unterschiede derselben Zeit, als einer erfülleten, oder leeren Zeit.“ (Kant 1976: A 143)

Das transzendentale Schema des Zeitinhalts müsste also im ersten Moment seiner Darstellung jenen Zeitabschnitt ausfüllen, in dem es einen unbestimmten aktuellen, reinen und intensiven Inhalt gibt, der wiederum eine unbestimmte Anzahl aktueller intensiver Zeiteinheiten umfasst. Eine extensive Raum- und Zeiteinheit hat eine Größe im Sinne potentieller Abschnitte, jedoch keine Extensität im Sinne einer Aneinanderreihung aktueller Abschnitte. Es klingt paradox, aber dank der indefiniten Theorie des Kontinuums kann eine extensive (Zeit-)Einheit (im kategorematischen Sinne) neben einer aktuellen nicht-extensiven Größe auch eine potentielle extensive Größe (im synkategorematischen Sinne) besitzen. Die Annahme, dass eine extensive Zeiteinheit nicht leer sei, bedeutet nichts anderes, als dass sie eine unbekannte Anzahl reiner intensiver Einheiten beinhaltet - deswegen ist die extensive Einheit ausgefüllt. Das zweite Moment des transzendentalen Schemas des Zeitinhalts bezieht sich auf die Negation (die Abwesenheit) aktueller intensiver Zeiteinheiten in der extensiven Zeiteinheit. Die Rede ist schlicht von einem Abschnitt, in dem kein aktueller intensiver Inhalt besteht (existiert, ist). Das dritte konstitutive Moment des transzendentalen Schemas des Zeitinhalts bezieht sich auf die Limitierung (Begrenzung) der extensiven Zeiteinheit durch ihre Unvollkommenheit, sowie die Limitierung ihres intensiven Inhalts, d. h. der intensiven Zeiteinheiten (ihrer Zusammenschlüsse und Verstärkungen). ${ }^{12}$ Auf der Basis des transzendentalen Schemas des Zeitinhalts wird eine reine Synthese intensiver Raumempfindungen geleistet. Kategorien der Qualität sind transponierte transzendentale Schemata des Zeitinhalts. Die Kategorien der Realität, Negation und Limitation kommen vor als isolierte Momente des transzendentalen Schemas des Zeitinhalts (das zweite mathematische Postulat a priori der reinen Einbildungskraft), das für die Tätigkeit der Einbildungskraft unerlässlich ist, vermittelst welcher sinnlich gegebene Erscheinungen und Wahrnehmungen synthetisiert werden. Daraus lässt sich nicht nur der Schluss ziehen, dass alle Erscheinungen Zeitinhalte darstellen, sondern auch der weitere, dass sie als Gegenstände der Wahrnehmung intensive Größen sind, die durch den Zusammenschluss einer gleichartigen Vielheit im Zeitinhalt erzeugt werden.

Heidegger ist der Ansicht, dass es Kant durch den Nachweis des Prinzips der „Antizipationen der Wahrnehmung“ gelungen ist, mittels der Auslegung der Gegenständlichkeit des Gegenstands das Gegebene, der Empfindung entsprechend, darzulegen. Als unerlässliche Bedingung für ein richtiges Verständnis der Empfindung analysiert er den Realitätsbegriff (vgl. Heidegger 1984: 214). Dasjenige, was an (empirischen) Gegenständen in Form von Erscheinungen der Empfindung entspricht (was diese auslöst), führt Kant als transzendentale Materie aller Gegenstände auf Dinge an sich zurück (Sachheit = Realität). ${ }^{13}$ Heideggers Vorlesung beginnt mit dem Hinweis darauf, dass sich die Realität von der Wirklichkeit und der Existenz unterscheidet; dass „Realität“ von realitas abgeleitet wird, während realis das bezeichnet, was der res (der Sache) zugeordnet werden kann. So bezeichnet z. B. ,das Reale“ (realis), das der res (der Sache) angehört, häufig „,das Ganze dieser Wesensbestimmung einer 
Sache oder die einzelnen Bestandstücke derselben“, z. B. die Ausdehnung eines natürlichen Körpers, seine Schwere, Dichtigkeit, Widerstandskraft usw. (vgl. Heidegger 1984: 215). Die Eigenschaften, die den Dingen als „real“ angehören, bedingen jedoch nicht zugleich die tatsächliche (wirkliche) oder auch nur potentielle Existenz der Sache. ${ }^{14}$ Der kritische und transzendentale Realitätsbegriff (realitas phaenomenon), das Reale in Erscheinungen, ist der erste Inhalt, der die Leere des Raums und der Zeit einnimmt, um die Entstehung der sinnlich gegebenen Erscheinungen zu ermöglichen. Von der Wahrnehmung (die stets empirisch ist, da es keine „reine Wahrneh-mung“ gibt) zur reinen Anschauung (von Raum und Zeit) ist eine stufenweise Wandlung möglich, nachdem sich das Reale des empirischen Bewusstseins vollkommen auflöst und nur die formale Anschauung der in Raum und Zeit gegebenen (sinnlichen) Mannigfaltigkeit übrig bleibt, bzw. nachdem die Synthesis der Produktion der Größe einer Wahrnehmung von ihrem Anfang an möglich ist, d. h. von der reinen Anschauung bis zu jeder beliebigen Größe dieser Wahrnehmung. ${ }^{15}$ Unabhängig von der Wahrnehmung kann die Empfindung keine objektive Vorstellung sein, da sie weder die Anschauung des Raums noch der Zeit beinhaltet und ihr daher keine Extension zugeschrieben werden kann. Es gehört ihr jedoch eine Größe auf Grund der Synthese der Apprehension, in der die Wahrnehmung (in einem bestimmten Zeitintervall) von null bis zu einer bestimmten Größe steigen kann. Intensive Größe bedeutet auch nichts anderes als einen bestimmten Grad an Einfluss auf die Sinne. Die extensive Größe aus den „Axiomen der Anschauung“ (aus dem ersten Grundsatz des reinen Verstandes) definiert Heidegger als quantitas, die Maßeinheit

12

Die transzendentale Einbildungskraft limitiert (begrenzt) durch ihre eigene (Selbst-)Tätigkeit den Inhalt intensiver Zeiteinheiten. Die Frage nach den Schemata der Qualitätskategorie wird durch die Angabe von zeitlichen Bestimmungen in der Produktion der Einbildungskraft beantwortet. Die Erfüllung der Zeit wäre dann also - wie wir bereits gesagt haben - ein Schema der Realitätskategorie, während die Abwesenheit (Leere) der Zeit ein Schema der Negationskategorie repräsentieren würde.

13

Dies entspricht der Formulierung aus A 143, in der die Realität dem Realen in den ,Antizipationen der Wahrnehmung" entspricht bzw. der realitas phaenomenon aus A 166 , wo „das Reale“ (realitas phaenomenon) mit der transzendentalen Materie (vielleicht besser: Inhalt) und mit der Sachheit empirischer Gegenstände als Erscheinungen gleichgesetzt wird. Fast dieselbe Haltung lässt sich in der Erörterung der ,Antizipationen der Wahrnehmung" erkennen, in der die Realität als realitas phaenomenon bezeichnet wird, wobei der germanisierte, aus dem Lateinischen stammende Ausdruck ,korrespondiert“" den deutschen Ausdruck ,entspricht“ ersetzt: „Was nun in der empirischen Anschauung der Empfindung korrespondiert, ist Realität (realitas phaenomenon) (...).“(Kant 1976: A 168)
14

Die Tatsache, dass ein Bett eine bestimmte Materialität, Extension, Farbe und Festigkeit aufweist, bedeutet nicht, dass dieses Bett de facto auch existiert, sondern nur, dass ihm bestimmte Eigenschaften zugeordnet werden können. Die Realität beantwortet nicht die Frage, ob dieses Bett existiert, sondern was eine bestimmte Sache ist. ,Wirklichkeit unterscheiden wir gegen Möglichkeit und gegen Notwendigkeit; alle drei Kategorien werden bei Kant zusammengefaßt unter den Titel Modalität. Daraus, daß in dieser Gruppe ,Realität' sich nicht findet, ist zu ersehen, daß Realität nicht Wirklichkeit bedeutet.“" (Heidegger 1984: 215-216) Kants Beispiel mit den ,hundert Talern“, egal ob sie möglich oder wirklich sind, lässt keine Schlussfolgerung bezüglich ihrer Realität zu. Die Kategorie der Qualität (Realität) sollte also nicht mit den Modalitätskategorien verwechselt werden (Möglichkeit, Dasein, Notwendigkeit).

15

In seiner Studie Kants theoretische Philosophie geht Arsenijević davon aus, dass der Unterschied im Hinblick auf die Qualität nur mithilfe des Unterschieds im Zusammenhang mit dem Raum festgestellt, jedoch nicht auf diesen reduziert werden kann. Außer dem Unterschied bezüglich der mathematischen Aufteilung des Raums gibt es nämlich auch den realen Unterschied dessen, was dieser Raum einnimmt (vgl. Arsenijević 1975: 47). 
einer aus mehreren Teilen bestehenden Menge. ${ }^{16}$ Die intensive Größe aus den „Antizipationen der Wahrnehmung“ (aus dem zweiten Grundsatz des reinen Verstandes) ist ebenfalls die quantitas einer qualitas, von etwas Realem (vgl. Heidegger 1984: 217-218). Jede Größe quantitas stellt die Einheit einer Mannigfaltigkeit dar. Bei intensiven Größen äußert sich diese Mannigfaltigkeit jedoch so, „daß ein Intensives der Negation - bis zur Null - angenähert wird“ (Heidegger 1984: 218). Die transzendentale Analyse findet zwei Arten von Unterschieden am Gegenstand vor, der erkannt wird: extensive und intensive Unterschiede bzw. Unterschiede hinsichtlich des Orts (und folglich auch der Größe und Form) im Raum und Unterschiede hinsichtlich der Erfülltheit der Zeit bei der Synthese der Apprehension dieser Gegenstände. ${ }^{17}$ Obwohl sich die Kategorien der Qualität nicht auf extensive Bestimmungen des Gegenstands beziehen, erfolgt ihre transzendentale Deduktion derart, dass die Qualität im Raum erscheint, sodass das Schema dieser Kategorien die Erfüllung der Zeit bedeutet. Aber diese Erfüllung steht dabei in Verbindung mit der Produktion der Einbildungskraft im Raum. Die Aussage, dass sich Qualitäten im Raum befinden, ist wegen der Art und Weise, wie wir einzig Gegenstände erkennen können, plausibel, und nicht deswegen, weil sich der Begriff der Qualität auf etwas Intensives (Stärkeres/Schwächeres) bezöge anstatt auf etwas Extensives (Größeres/Kleineres). Die Qualitäten sind in der Empfindung gegeben, die qualitative Vielfalt dagegen im Raum durch die Synthese der Apprehension des produktiven Vermögens der transzendentalen Einbildungskraft. Nehmen wir z. B. ein „rotes Bett“ wahr, so bezieht sich unsere Empfindung auf das Rot, während sich unsere Wahrnehmung auf die Extension dieses roten Gegenstands bezieht, der „Bett“ genannt wird. Die Wahrnehmung ist die Wahrnehmung von etwas, das man fühlen kann, während die Empfindung das Fühlen von etwas ist, was wahrgenommen wird. Die Anschauung ist als Form eines inneren Sinnes wiederum eine Vorstellung, die jeglichem Akt des Denkens vorausgeht. Diese Anschauung ist eine sinnliche, keine intellektuelle Anschauung.

Neben der räumlich-zeitlichen Bestimmtheit beinhalten sinnlich gegebene Erscheinungen auch dasjenige, was Heidegger ,das Andrängende“ nennt, dasjenige ,was uns angeht und aufliegt und den raumzeitlichen Bereich besetzt" (Heidegger 1984: 220). Das ist die sogenannte transzendentale Materie oder, treffender gesagt, der transzendentale Inhalt als offener Bereich der realitas phaenomenon, der als Spanne zwischen Erfüllung und Leere (Realität und Negation) definiert werden kann. ${ }^{18}$ Das Axiom der Anschauung und die Antizipationen der Wahrnehmung sind mathematische Grundsätze des reinen Verstandes, ,diejenigen, die die Möglichkeit einer Anwendung von Mathematik auf Gegenstände metaphysisch begründen“ (Heidegger 1984: 225). Analog zu diesen Grundsätzen haben sowohl die transzendentalen Schemata der Zeitreihe als auch diejenigen des Zeitinhalts den Charakter von mathematischen Schemata. Die reinen Verstandesbegriffe, die in den apriorischen Bereich fallen, wären ,leer“", wenn ihnen nicht die sinnliche Mannigfaltigkeit a priori zur Verfügung stünde, denn diese Mannigfaltigkeit ,füllt“ die Begriffe mit ihrem Inhalt. ${ }^{19}$ Die rein sinnliche Mannigfaltigkeit (also diejenige, die $a$ priori gegeben ist) ist eine Form unserer Anschauung (die Rede ist von der Mannigfaltigkeit der räumlich-zeitlichen Beziehungen). Die Zeit ist also eine Form der Anschauung, zugleich jedoch auch eine formale Anschauung (vgl. Kant 1976: B 161). Als Form der Anschauung ist die Zeit (wie auch der Raum) eine Form der a priori gegebenen sinnlichen Mannigfaltigkeit, aber als formale Anschauung enthält die Zeit auch die Einheit dieser a priori gegebenen rein sinnlichen Mannigfaltigkeit. ${ }^{20}$ Die Anschauung existiert nur, 
wenn uns ein Gegenstand (als Erscheinung) zur Verfügung steht, d. h., wenn er unsere Sinne affiziert, wobei der Gegenstand das Affizierende, die Sinne dagegen das Affizierte sind. Die Einheit der formalen Anschauung ist keine kategoriale Einheit (der Quantität), sondern der Nexus des Vorstellungsvermögens bezüglich der Mannigfaltigkeit, die nicht auf empirische Art von den Sinnen aufgenommen werden kann, sondern a priori, als Synthese zum Zuge kommt. Die Vorstellung von der synthetischen Einheit der Mannigfaltigkeit kann allerdings nicht aus diesem Nexus als Synthese dieser Mannigfaltigkeit entstehen, sondern diese Einheit ist a priori gegeben und macht diesen Begriff des Nexus möglich, wenn er neben der (hinzukommenden) Vorstellung der Mannigfaltigkeit besteht. Wenn die Synthese der reinen Einbildungskraft früher als die Einheit der transzendentalen Apperzeption entsteht (also vor ihr), dann ist nicht die Rede von einem zeitlichen, sondern von einem logischen Vorausgehen. Im Einklang mit dem Prinzip des logischen Vorrangs würden die Erkenntniskonstituenzien mit der (Einheit der) formalen Anschauung beginnen und sich über die Synthese der transzendentalen Einbildungskraft bis zur begrifflichen Einheit der Kategorien und der Einheit der transzendentalen Apperzeption erstrecken.

Abschließend möchten wir hinzufügen, dass einer der Haupteinwände Heideggers gegen die kantische Theorie der Zeit darin besteht, dass Kant nicht „ausreichend“ den Zusammenhang zwischen der Zeitlichkeit und dem Sein thematisiert hat. Nachdem die Zeitlichkeit nämlich eine Bedingung für das Verstehen des Seins darstellt, ist sie auch Voraussetzung für die Explikation der Problematik der Temporalität und ihrer ekstatisch-horizontalen Struktur. Kants Erörterung der mathematischen und dynamischen transzendentalen

16

Quantitative Größenunterschiede lassen sich auf den Unterschied zwischen extensiv und intensiv zurückführen und wenn eine Größe nicht nur ein Gegenstand, sondern auch ein Produkt der Wahrnehmung ist, dann unterscheiden sich auch extensive und intensive Wahrnehmung, sowie die extensiven und in tensiven Empfindungen als deren Einheiten.

17

Arsenijević hat recht, wenn er sagt, dass wir (als Subjekte der Erkenntnis) in unserem Blickfeld verschiedene Qualitäten und die Negation irgendeiner Qualität vorfinden, nicht aber die Negation an sich. „Die Qualität hängt in jedem Fall insoweit von der Apperzeption ab, als sie begrifflich als Qualität definiert ist (z. B. visuelle Qualität, Farbe, Röte usw.), sie ist jedoch nicht als solche abhängig, d. h. als dasjenige, auf das sich diese Bestimmung am gegebenen Gegenstand bezieht." (Arsenijević 1975: 51)

18

Heidegger schließt, ausgehend vom Kants Text, dass die Empfindung als das Reale über eine Quantität verfügt, die zahlenmäßig durch eine sukzessive Addition von Einheiten bestimmt werden kann (wenn einer Einheit eine weitere hinzugefügt wird). Der Freiburger Philosoph wäre sich selber nicht treu, wenn er keine Änderung des zweiten Grundsatzes des reinen Verstandes vorgeschlagen hätte (Antizipationen der Wahrnehmung), welche wie folgt lautet: „In allen Erscheinungen hat die Empfindung, und d. h. zuvor das Reale, das sie (acc.) als ein Gegenständliches sich zeigen läßt, eine intensive Größe.“ (Heidegger 1984: 222)

19

In der reinen Mannigfaltigkeit als Form unserer Anschauung a priori sieht Todorović die ,Mannigfaltigkeit räumlicher und zeitlicher Beziehungen, die so den Stoff der reinen Begriffe ausmachen, wobei gleichzeitig die Bedingungen erhalten bleiben, unter denen unser Gemüt überhaupt die Vorstellungen aufnimmt - sie bleiben die Bedingungen seiner Rezeptivität.“ (Todorović 2004: 147)

20

Todorović geht davon aus, dass Raum und Zeit - als Formen der Anschauung und als Bedingung der Rezeptivität unseres Gemüts als konstituierendes Element der Gegenständlichkeit der Gegenstände unserer Vorstellungen den Begriff als transzendentale Bedingung seines Gegenstands affizieren müssen, während die Struktur des Selbstbewusstseins bzw. der transzendentalen Apperzeption die Grundlage des Begriffs bildet (vgl. Todorović 2004: 147) 
Schemata bezieht sich auf die Zeit, nicht jedoch auf die Zeitlichkeit. Es fehlt ihr daher die Problematik der Temporalität, welche neben der Zeitlichkeit des Daseins auch Fragen der Intentionalität und Transzendenz miteinschließt. Heidegger ist der Ansicht, dass sich ,[e]rst aus der Temporalität des Seinsverständnisses (...) aufklären" lässt, ,warum die ontologischen Bestimmungen des Seins den Charakter der Apriorität haben“ (Heidegger 1975: 462).

\title{
Literatur
}

Arsenijević, M. (1975): „Kantova teorijska filozofija“, Filozofske studije 6 (1975), S. 9-114.

Heidegger, M. (1993): Sein und Zeit, Max Niemeyer Verlag, Tübingen.

Heidegger, M. (1984): Die Frage nach dem Ding. Zu Kants Lehre von den transzendentalen Grundsätzen, Gesamtausgabe. II. Abteilung: Vorlesungen Wintersemester 1935/36, Band 41, Vittorio Klostermann, Frankfurt am Main.

Heidegger, M. (1977): Phänomenologische Interpretation von Kants Kritik der reinen Vernunft, Gesamtausgabe. II. Abteilung: Vorlesungen Wintersemester 1927/28, Band 25, Vittorio Klostermann, Frankfurt am Main.

Heidegger, M. (1976): Logik. Die Frage nach der Wahrheit, Gesamtausgabe. II. Abteilung: Vorlesungen Wintersemester 1925/26, Band 21, Vittorio Klostermann, Frankfurt am Main.

Heidegger, M. (1975): Die Grundprobleme der Phänomenologie, Gesamtausgabe. II. Abteilung: Vorlesungen Sommersemester 1927, Band 24, Vittorio Klostermann, Frankfurt am Main.

Heidegger, M. (1973): Kant und das Problem der Metaphysik, Vittorio Klostermann, Frankfurt am Main.

Kant, I. (1976): Kritik der reinen Vernunft, Felix Meiner Verlag, Hamburg.

Todorović, M. (2004): „O jednoj Kantovoj reči“, Arhe 1 (2004) 2, S. 133-155.

\section{Biljana Radovanović, Goran Ružić}

\section{Heideggerova radikalizacija Kantova učenja o matematičkim shemama i vremenu}

\begin{abstract}
Sažetak
Osnovni motiv Heideggerova tumačenja Kantove teorijske filozofije sastoji se u tome da se pokaže da Kantovo učenje o shematizmu i vremenu predstavlja tek pred-stupanj problematike temporalnosti. U tom kontekstu analizirat ćemo Heideggerovu tezu o transcendentalnoj uobrazilji kao »izvoru« vremena, odnosno matematičke sheme vremenskog niza i vremenskog sadržaja. S obzirom na to da su ove sheme nužnim načinom povezane s kategorijama kvantitete i kvalitete, kao is načelima (sintetičkim stavovima a priori) čistog razuma koja je Kant formulirao kao »aksiome zrenja« $i$ »anticipacije opažaja«, jasno je da je time naš interpretativni zadatak postao složeniji. Paralelno s navedenim, morali smo dovesti u vezu jedinstvo vremena (kao formalnog zora) s jedinstvom praosnovne apercepcije (strukture samosvijesti), kao i osjetilno danu mnogostrukost s vremenom kao formom čistog osjetilnog zora. Usporedna analiza A i B izdanja Kantove Kritike čistog uma omogućila nam je, preko Heideggerovih predavanja $i$ studija, da unutar Kantova teksta sagledamo mogućnosti »ontologizirane« i fenomenološki usmjerene interpretacije i tumačenja osnovnih postavki Kantove »teorije spoznajnog subjekta«.
\end{abstract}

\section{Ključne riječi}

transcendentalna filozofija, shema, prostor, vrijeme, zor, opažaj, razum, apercepcija 
Biljana Radovanović, Goran Ružić

\title{
Heidegger's Radicalisation of Kant's Teaching on Mathematical Schemata and Time
}

\begin{abstract}
The basic motive of Heidegger's interpretation of Kant's theoretical philosophy consists of an intention to show that Kant's teaching on schematism and time represents a pre-phase of the problem of temporality. In that context, we will analyse Heidegger's thesis on transcendental imagination as the "source" of time, that is, of the mathematical schemata of the timeline and the content of time. Considering that these schemata are by necessity connected to the categories of quantity and quality, as well as with principles (synthetic judgments a priori) of pure reason, which Kant formulated as the "axioms of intuition" and "anticipation of perception", it is clear that this makes our interpretative task more complicated. In parallel, we had to make a connection between the unity of time (as formal intuition) and the unity of fundamental aperception (structure of consciousness), as well as the perceptual multiplicity with the time as the pure form of intuition. Comparative analysis of $A$ and $B$ editions of Kant's Critique of Pure Reason allowed us to, by using Heidegger's lectures and studies, consider within Kant's text the possibility of "ontologised" and phenomenologically focused interpretation of fundamental assumptions of Kant's "theory of knowing subject".
\end{abstract}

Keywords

transcendental philosophy, scheme, space, time, intuition, perception, reason, aperception

\section{Biljana Radovanović, Goran Ružić}

\section{La radicalisation heideggérienne des enseignements de Kant sur les schèmes mathématiques et le temps}

\begin{abstract}
Résumé
La raison principale de l'interprétation heideggérienne de la théorie philosophique de Kant réside dans l'intention de montrer que l'enseignement kantien sur le schématisme et le temps ne présente qu'un pré-niveau de la problématique de la temporalité. Dans ce contexte, nous analyserons la thèse heideggérienne de l'imagination transcendantale en tant que la " source »du temps, à savoir les schèmes mathématiques de la succession temporelle et du contenu temporel. Étant donné que ces schèmes sont de manière nécessaire liées aux catégories de la quantité et de la qualité, autant qu'elles le sont aux principes (à travers les jugements synthétiques a priori) de la raison pure que Kant formule en tant qu' "axiomes de l'intuition » et " anticipations de la perception ", il est clair qu'ainsi notre devoir d'interprétation est devenu plus complexe. En parallèle avec ce qui vient d'être mentionné, nous avons été contraints de mettre en lien l'unité du temps (en tant qu'intuition formelle) avec l'unité originelle de l'aperception (structures de la conscience de soi), mais également la multiplicité du donné sensible avec le temps et la forme de l'intuition pure sensible. L'analyse comparative des éditions $A$ et $B$ de la Critique de la raison pure nous a permis, à travers les cours et les études de Heidegger, de considérer à l'intérieur des textes kantiens les possibilités pour une interprétation à tendance "ontologiseante » et phénoménologique, et d'interpréter les données principales « de la théorie du sujet connaissant ».
\end{abstract}

\section{Mots-clés}

philosophie transcendantale, schème, espace, temps, intuition, perception, entendement, aperception 